\title{
ON A POSSIBLE MECHANISM OF PULSAR RADIATION
}

\author{
A. Z. Kazbegi, G. Z. Machabeli and G. I. Melikidze \\ Abastumani Astrophysical Observatory
}

At present there exist several well-grounded models of pulsar radiation which do not exactly coincide with each other [e.g. Ruderman and Sutherland (1975), Cheng and Ruderman (1980), Arons and Sharlemann (1979), Arons (1981)]. The creation of a dense, relativistic, electron-positron plasma in the polar regions of rotating neutron star magnetospheres is the point of similarity between these models. Surely the pulsar radiation should be generated in such a plasma. The plasma density near the stellar surface is $n_{\mathrm{p}} \simeq 10^{16}$ to $10^{17} \mathrm{~cm}^{-3}$, and the average Lorentz-factor of the particles is $\gamma_{\mathrm{p}}=3$ to 10 . The plasma is penetrated by the beam of "primary" electrons, extracted from the stellar surface and accelerated by the electric field. The beam parameters are as follows: $n_{\mathrm{b}}=7 \times 10^{-2} B_{0} P^{-1}$, where $P$ is the pulsar period and $B_{0}$ magnetic field at the stellar surface and $\gamma_{\mathrm{b}}=3 \times 10^{6}$ to $10^{7}$. The plasma flowing along the open magnetic field lines possesses an asymmetrical distribution function with a tail stretched out in the direction of positive momenta [e.g. Arons (1981)] having an average Lorentz-factor $\gamma_{\mathrm{t}} \simeq 10^{3}$ to $10^{4}$. All these parameters correspond to a "typical" pulsar $(P \approx 1 \mathrm{~s})$. Due to the strong magnetic field, the transverse components (with respect to the magnetic field $\mathbf{B}_{\mathbf{0}}$ ) of the particle momenta $p_{\perp}$ decay and the distribution function tends to be one-dimensional. The distribution functions of the electrons and positrons do not exactly coincide with each other and $\gamma_{+}-\gamma_{-}=\Delta \gamma \neq 0$, where $\gamma_{+}$and $\gamma_{-}$are the average Lorentz-factors of positrons and electrons, respectively.

Most probably the magnetic field near the pulsar has a complicated structure differing greatly from a dipole. Though at sufficiently large distances from the stellar surface up to the light cylinder the magnetic field can be considered as dipolar: $B=B_{0}\left(R_{0} / R\right)^{3}$ if $R_{0}<R<c / \Omega$, where $R_{0}=10^{6} \mathrm{~cm}$ is a neutron star radius and $\Omega$ is the pulsar angular velocity. The dependence of plasma density on distance is the same $n_{\alpha}=n_{0 \alpha}\left(R_{0} R\right)^{3}$, where the index "0" denotes values taken at the stellar surface and $\alpha$ corresponds to the plasma particle species (electrons, positrons, beam).

In our opinion only the maser emission mechanisms (Ginzburg and Zheleznyakov 1975) are well grounded [as opposed to e.g. the antenna mechanisms; for more details see Lominadze et al. (1986).] In this paper we give additional arguments confirming this opinion.

In order to consider the curvature of the magnetic field lines correctly, cylindric coordinates $x, r, \varphi$ will be used below. The $x$-axis is directed transverse to the plane of field line curvature, $r$ is the radial and $\varphi$ the azimuthal coordinate, respectively. The latter corresponds to the curvature of the field line (torsion is neglected and $\partial R_{\mathrm{B}} / \partial r=0$, where $R_{\mathrm{B}}$ is the radius of curvature of field liye). In such a geometry one has following integrals of motion: $\gamma, p_{x}-\omega_{B \alpha} r / c, p_{\varphi} r$. Here $p_{i}=v_{i} \gamma / c$ and $v_{i}$ are the components of the particle momentum and velocity, and $\omega_{B \alpha}=e_{\alpha} B / m c$. The particle distribution function should depend on the integrals of motion. Using the method of integration along the particle trajectory one obtains the components of the dielectrical permeability tensor:

$$
\begin{aligned}
& \epsilon_{x x}=1-\frac{1}{2} \sum_{\alpha} \frac{\omega_{p \alpha}^{2}}{\omega^{2}} \int \frac{d p_{\varphi}}{\gamma}\left(\left(\omega-k_{\varphi} v_{\varphi}-2 k_{\varphi} u_{\alpha}^{2} / c\right) A_{\alpha}^{+} f_{\alpha}+2 \omega \frac{u_{\alpha}^{2}}{c^{2}} \frac{\gamma}{\Omega_{\alpha}^{0}} \frac{\partial f_{\alpha}}{\partial \gamma}\right) \\
& \epsilon_{r r}=1-\frac{1}{2} \sum_{\alpha} \frac{\omega_{p \alpha}^{2}}{\omega^{2}} \int \frac{d p_{\varphi}}{\gamma}\left(\omega-k_{\varphi} v_{\varphi}-k_{x} u_{\alpha}\right) A_{\alpha}^{+} f_{\alpha} \\
& \epsilon_{\varphi \varphi}=1+\sum_{\alpha} \frac{\omega_{p \alpha}^{2}}{\omega^{2}} \int d p_{\varphi} \frac{v_{\varphi}^{2}}{c^{2} \Omega_{\alpha}^{0}} \frac{\partial f_{\alpha}}{\partial \gamma} \\
& \epsilon_{x r}=-\frac{i}{2} \sum_{\alpha} \frac{\omega_{p \alpha}^{2}}{\omega^{2}} \int \frac{d p_{\varphi}}{\gamma}\left(\omega-k_{\varphi} v_{\varphi}-k_{x} u_{\alpha}\right) A_{\alpha}^{-} f_{\alpha} \\
& \epsilon_{r x}=-\frac{i}{2} \sum_{\alpha} \frac{\omega_{p \alpha}^{2}}{\omega^{2}} \int \frac{d p_{\varphi}}{\gamma}\left(\omega-k_{\varphi} v_{\varphi}-2 k_{\varphi} u_{\alpha}^{2} / c\right) A_{\alpha}^{+} f_{\alpha} ; \\
& \epsilon_{x \varphi}=-\frac{1}{2} \sum_{\alpha} \frac{\omega_{p \alpha}^{2}}{\omega^{2} c} \int \frac{d p_{\varphi}}{\gamma} v_{\varphi}\left(\left(k_{x} c A_{\alpha}^{+}+i k_{r} c A_{\alpha}^{-}\right) f_{\alpha}+2 \omega \frac{u_{\alpha}}{c} \frac{\gamma}{\Omega_{\alpha}^{0}} \frac{\partial f_{\alpha}}{\partial \gamma}\right) \\
& \epsilon_{\varphi x}=-\frac{1}{2} \sum_{\alpha} \frac{\omega_{p \alpha}^{2}}{\omega^{2} c} \int \frac{d p_{\varphi}}{\gamma} v_{\varphi}\left(\left[\left(k_{x} c-2 k_{\varphi} u_{\alpha}\right) A_{\alpha}^{+}-i k_{r} c A_{\alpha}^{-}\right] f_{\alpha}+2 \omega \frac{u_{\alpha}}{c} \frac{\gamma}{\Omega_{\alpha}^{0}} \frac{\partial f_{\alpha}}{\partial \gamma}\right)
\end{aligned}
$$




$$
\begin{aligned}
& \epsilon_{r \varphi}=-\frac{1}{2} \sum_{\alpha} \frac{\omega_{p \alpha}^{2}}{\omega^{2} c} \int \frac{d p_{\varphi}}{\gamma} v_{\varphi}\left(\left[k_{r} c A_{\alpha}^{+}-i\left(k_{x} c-2 k_{\varphi} u_{\alpha}\right) A_{\alpha}^{-}\right] f_{\alpha}\right) \\
& \epsilon_{\varphi r}=-\frac{1}{2} \sum_{\alpha} \frac{\omega_{p \alpha}^{2}}{\omega^{2} c} \int \frac{d p_{\varphi}}{\gamma} v_{\varphi}\left(\left[k_{r} c A_{\alpha}^{+}+i\left(k_{x} c-2 k_{\varphi} u_{\alpha}\right) A_{\alpha}^{-}\right] f_{\alpha}\right)
\end{aligned}
$$

where

$$
\begin{array}{ll}
A_{\alpha}^{+}=\left(\frac{1}{\Omega_{\alpha}^{+}}+\frac{1}{\Omega_{\alpha}^{-}}\right) ; \quad \Omega_{\alpha}^{ \pm}=\omega-k_{\varphi} v_{\varphi}-k_{x} u_{\alpha} \pm \omega_{B \alpha} \gamma^{-1} ; \quad \omega_{p \alpha}^{2}=4 \pi e^{2} n_{p \alpha} / m ; \\
A_{\alpha}^{-}=\left(\frac{1}{\Omega_{\alpha}^{-}}-\frac{1}{\Omega_{\alpha}^{+}}\right) ; \quad \Omega_{\alpha}^{0}=\omega-k_{\varphi} v_{\varphi}-k_{x} u_{\alpha} ; & f_{\alpha}=\int d p_{\perp} p_{\perp} F_{\alpha} .
\end{array}
$$

$F_{\alpha}$ is the particle distribution function; $p_{\perp}=\sqrt{p_{r}^{2}+\left(p_{x}-u \gamma / c\right)^{2}}$; the sum over $\alpha$ is taken over the particle species and the integration is from $-\infty$ to $+\infty ; u_{\alpha}=v_{\varphi} p_{\varphi} c / \omega_{B \alpha} R_{B}$ is the particle drift velocity caused by the weak inhomogeneity (curvature) of the magnetic field and is directed along the $x$-axis for positrons (hereafter for values without the subscript $\alpha$, the sign of the charge is assumed to be positive.). For the particles of the bulk plasma the drift velocity $u$ tends to be zero. Hence if one assumes $u_{\alpha}=0$ eq. (1) reduces to the standard form which we designate as $\epsilon_{i j}^{\mathrm{p}}$. In general, however, $\epsilon_{i j}=\epsilon_{i j}^{\mathrm{p}}+\epsilon_{i j}^{\mathrm{b}}$, where $\epsilon_{i j}$ is described by eq.(1), $\epsilon_{i j}^{\mathrm{p}}$ corresponds to an electron-positron plasma and $\epsilon_{i j}^{\mathrm{b}}$ corresponds to the beam.

For propagation properties the actual drift velocity is not important. In the electron-positron plasma described by $\epsilon_{i j}^{\mathrm{p}}$ there exist two types of waves: the purely transverse electromagnetic $t$-wave and the potential-nonpotential lt-wave.

If the angle $\theta=k_{\perp} / k_{\varphi}$, where $k_{\perp}=\sqrt{k_{x}^{2}+k_{r}^{2}}$, satisfies condition $\theta^{2} \gg \theta_{0}^{2} \equiv \Delta \gamma \omega / 8 \omega_{b} \gamma_{\mathrm{p}}^{4}$, these waves are linearly polarized. The electric vector $E^{t}$ of the $t$-waves is directed transverse to the plane containing both the magnetic field line and the wave vector. The electric vector $E^{\text {lt }}$ of the lt-waves lies in the plane of $\boldsymbol{k}$ and $B$. The spectra of the waves are as follows

$$
\begin{aligned}
\omega^{\mathrm{t}} & =k c(1-\delta), \\
\omega^{\mathrm{tt}} & =k_{\varphi} c\left(1-k_{\perp}^{2} c^{2} / 8 \omega_{\mathrm{p}}^{2} \gamma_{\mathrm{p}}\right)
\end{aligned}
$$

where

$$
\delta=\omega_{\mathrm{p}}^{2} / 4 \omega_{B}^{2} \gamma_{\mathrm{p}}^{3}
$$

Note that eqs.(2) and (3) are obtained for $\omega<\omega_{b} \gamma_{p}$ and that eq.(3) describes only the low frequency branch of the lt-waves. The phase velocity of the high frequency branch exceeds the speed of light and cannot interact with particles. In the opposite case when $\theta^{2} \ll \theta_{0}^{2}$, then both the $t$ and the lt waves coincide and turn into an electromagnetic circularly polarized wave with the spectrum given by eq. (2). The electromagnetic wave spectra are near the "vacuum" spectra $(\omega=k c)$, so they can leave the magnetosphere without transformation.

Sagdeev and Shafranov (1960) were the first to point out the existence of the cyclotron instability in a plasma with an anisotropic distribution function (in our case $p_{\|} \gg p_{\perp}$ ). Let us investigate the possibility of $t$-wave generation by means of the cyclotron resonance, the condition of which is

$$
\omega-k_{\varphi} c-k_{x} u_{\alpha} \pm \frac{\omega_{B \alpha}}{\gamma}=0
$$

Substituting eq.(2) and the expressions $k=k_{\varphi}\left(1+k_{\perp}^{2} / 2 k_{\varphi}^{2}\right)$ and $v_{\varphi}=c\left(1-1 / 2 \gamma^{2}-u^{2} / 2 c^{2}\right)$ in eq.(4) one obtains

$$
\frac{k_{r}^{2}}{2 k_{\varphi}^{2}}+\frac{1}{2 \gamma_{\text {res }}^{2}}+\frac{1}{2}\left(\frac{k_{x}}{k_{\varphi}}-\frac{u_{\alpha}}{c}\right)^{2}-\delta=\mp \frac{\omega_{\mathrm{b}}}{k_{\varphi} c \gamma_{\mathrm{res}}}
$$

As was shown by Machabeli and Usov (1979) the $t$ waves are excited in the pulsar magnetosphere only by the anomalous Doppler effect [upper sign of eq.(5)]. The lower sign of eq.(5) corresponds to wave damping on the particles of the bulk of plasma $\left(\gamma_{\text {res }} \sim \gamma_{p}\right)$. It follows that for frequencies $\omega<2 \omega_{b} \gamma_{p}$ damping of the magnetosphere plasma does not occur.

For the development of the cyclotron instability it is necessary to satisfy the condition

$$
R=R_{0}\left(\frac{\gamma_{\mathrm{p}}^{2}}{\gamma_{\mathrm{res}}}\right)^{1 / 3}\left(\frac{\omega_{B o}}{\omega_{\mathrm{po}}}\right)^{2 / 3} \leq \frac{c}{\Omega},
$$


where $R$ is the distance between the neutron-star center and the region in which the instability develops. Pulsars with dipole magnetic fields may have difficulty satisfying the condition of eq.(6) due to their very large values of $\gamma_{p} \geq 10^{2}$ to $10^{3}$ (Cheng and Ruderman 1980), while eq.(6) gives $\gamma_{p}<10$. It seems more probable that the magnetic field of a neutron star near the stellar surface differs greatly from dipolar, and the curvature of the field lines $R_{\mathrm{b}}$ is of the order of $R_{0}$ (Ruderman and Sutherland 1975). Observational data on pulsars (Davies et al. 1984) and accreting neutron stars in binary systems [like Her X1 (Pines 1980)] testify to the above assumption. In a magnetic field with $R_{\mathrm{B}} \sim R_{0}$ one obtains $\gamma_{p}<10$. Consequently for more complex magnetic field configurations the region of cyclotron instability development may be inside the light cylinder both for the beam of the primary particles and for the high energy tail of the plasma particles.

The resonance condition [eq.(5) with the upper sign] is satisfied only if $\delta \gg 1 / 2 \gamma_{\text {res }}^{2}$ and $k_{\perp} \rightarrow 0$. Thus the waves are excited in a very narrow range of angles $\theta$. The growth rate of cyclotron instability is

$$
\begin{gathered}
\Gamma=\pi \frac{\omega_{\mathrm{p} \text { res }}^{2}}{\omega_{0} \gamma_{\mathrm{t}}} \quad \text { if } \quad \frac{1}{2} \frac{u^{2}}{c^{2}} \ll \delta, \\
\text { and } \quad \Gamma=\pi \frac{\omega_{\mathrm{p} \text { res }}^{2}}{2 \omega_{0} \gamma_{\mathrm{t}}} \frac{u^{2}}{\delta c^{2}} \quad \text { if } \quad \frac{1}{2} \frac{u^{2}}{c^{2}} \gg \delta,
\end{gathered}
$$

and the resonant frequency is defined as $\omega_{0} \approx \omega_{\mathrm{b}} / \delta \gamma_{\text {res }}\left(\gamma_{\mathrm{t}}\right.$ is the thermal spread of the resonant particles).

Note that eqs. (7) and (8) are valid only if the resonance width is more than the growth rate (the so-called condition of the kinetic approximation). This leads to the following limitation $\Gamma \ll \omega_{b} \gamma_{t} / \gamma_{\text {res }}^{2}$. From this and eqs.(7) and (8) it follows that for the particles of the tail the excitation of $t$ waves is possible at distances $R \simeq 10^{8} \mathrm{~cm}$ from the center of the pulsar. However for particles of the primary beam the distance is $R \simeq 10^{9} \mathrm{~cm}$.

The interaction of the excited $t$ waves with the beam particles brings about the quasilinear diffusion of particles in momentum space. As a result the beam particles acquire non-zero pitch angles $\psi$ $\left(\tan \psi=p_{\perp} / p_{\varphi}\right)$. Besides, the particles in the inhomogeneous magnetic field $B(R)$ drift with velocity $u_{x}$. As was shown by Machabeli and Usov (1979), in the central parts of the magnetosphere the value of $\psi \propto R_{\mathrm{b}}^{2}$ and so may exceed $u_{x}$. In this case the resonance condition $\omega_{0}-k_{\varphi} v_{\varphi}-k_{x} u_{x}=0$ due to the fact that $v_{\varphi} / c=1-1 / 2 \gamma_{\text {res }}^{2}-\psi^{2} / 2-u_{x}^{2} / 2 c^{2}$ will take the following form

$$
\left(\frac{k_{\perp}}{k_{\varphi}}\right)^{2}+\psi^{2} \simeq 2 \delta .
$$

This type of Cherenkov resonance can be easily fulfilled. Let us consider the possibility of $t$-wave excitation by the beam particles by means of Cherenkov resonance (Kazbegi, Machabeli, and Melikidze 1987b). Solving the imaginary part of the dispersion relation and using the relativistic Maxwell distribution function $\exp \left[-\left(\gamma-\gamma_{b}\right)^{2} / \gamma_{t}^{2}\right]$ for the beam, we obtain the growth rate of the $t$ waves

$$
\frac{\Gamma}{\omega_{0}}=\frac{\pi}{8}\left(\frac{\omega_{\mathrm{b}}}{\omega_{\mathrm{B}}}\right)^{2} \frac{k_{\perp}^{2}}{k^{2}} \gamma_{\mathrm{b}}\left(\frac{\gamma_{\perp 0}}{\gamma_{\mathrm{t}}}\right)^{2} \sim 10^{-5} \text { to } 10^{-6} .
$$

The condition of the kinetic approximation yields

$$
\frac{\Gamma}{\omega_{0}}<\psi^{2} \frac{\gamma_{t}}{\gamma_{b}} \sim 10^{-4} \text { to } 10^{-5}
$$

Comparison of eqs.(9)-(11) gives an estimate $\Gamma / \omega_{0} \sim \delta^{4 / 3}$, which shows the possibility of the instability developing at a distance $R \simeq 5 \times 10^{8}$ to $10^{9} \mathrm{~cm}$.

Substitution of the lt-wave spectrum (3) into the Cherenkov resonance condition yields

$$
\psi^{2}=\left(\frac{k_{\perp}}{k_{\varphi}}\right)^{2} \frac{\omega_{0}^{2}}{2 \omega_{\mathrm{p}}^{2} \gamma_{\mathrm{p}}},
$$

and the growth rate of the lt waves for $\left(\partial f_{\varphi} / \partial p_{\varphi}\right)>0$ is given by

$$
\frac{\Gamma}{\omega_{0}}=\pi\left(\frac{\omega_{\mathrm{b}}}{\omega_{0}}\right)^{2} \gamma_{\mathrm{b}}^{3}\left(\frac{k_{x}}{k_{\varphi}}\right)^{2} \frac{1}{\gamma_{\mathrm{t}}^{2} \gamma_{\perp 0}^{2}}
$$


The drift velocity $u_{x}$ of beam particles moving from the pulsar to the light cylinder increases since $u_{x} \propto\left(R / R_{0}\right)^{a}$, where $a \simeq 2$ relies on the dependence of $R_{\mathrm{B}}$ on $R$. In the region where $u_{x}>\psi$, the Cherenkov-resonance condition for the $t$ waves is

$$
\delta-\frac{1}{2} \frac{k_{r}^{2}}{k_{\varphi}^{2}}-\frac{1}{2}\left(\frac{u_{x}}{c}-\frac{k_{x}}{k_{\varphi}}\right)^{2}=0,
$$

and for the lt waves is

$$
\frac{u_{x}^{2}}{c^{2}}=\frac{k_{\perp}^{2}}{k_{\varphi}^{2}} \frac{\omega_{0}^{2}}{2 \omega_{\mathrm{p}}^{2} \gamma_{\mathrm{p}}}+2 \frac{k_{x}}{k_{\varphi}} \frac{u_{x}}{c}
$$

Making use of the eq.(1) one can obtain the growth rate for both the $t$ and lt waves

$$
\frac{\Gamma}{\omega_{0}} \simeq \pi \frac{\omega_{\mathrm{b}}^{2}}{\omega_{0}^{2}}\left(\frac{k_{r}}{k_{\varphi}}\right)^{2} \gamma_{\mathrm{b}}\left(\frac{\partial f_{\varphi}}{\partial p_{\varphi}}\right)_{p_{\varphi}=p_{\varphi}^{\mathrm{r}}}
$$

The kinetic instability condition for both types of waves is given by

$$
\frac{\Gamma}{\omega_{0}}<\left(\frac{u_{x}}{c}\right)^{2} \frac{\gamma_{t}}{\gamma_{b}}
$$

It can be seen from eqs.(16) and (17) that for typical pulsar parameters the drift motion causes $t$ and lt-wave excitation at distances $R \simeq 10^{9} \mathrm{~cm}$, corresponding to radio emission in the frequency range $\omega_{0} \simeq 10^{8}$ to $10^{10} \mathrm{~Hz}$.

Therefore, we conclude that wave excitation in the plasma of the pulsar magnetosphere is possible. The first possibility arises when the cyclotron instability develops due to resonant interaction between $t$ waves and both beam and tail particles.

The excited $t$ waves originate with $k_{\perp} \rightarrow 0$. We believe that this kind of excitation can explain the "core" type of pulsar emission (Rankin 1983a). It is worthwhile to emphasize that this is the most stable mechanism among those considered in this paper. The development of the cyclotron instability is associated with a quasilinear interaction of the waves with energetic particles. As a result beam particles obtain non-zero pitch angles $\left(p_{\perp} \neq 0\right.$ ). Additionally, at distances of about $R \simeq 10^{\circ} \mathrm{cm}$ (for a typical pulsar) the particle-drift motion caused by the magnetic field curvature becomes essential. In this case the Cherenkov-resonance condition can be satisfied in different parts of the magnetosphere: in the central part since $\psi>u_{x} / c$, and at the edges where $\psi<u_{x} / c$. In both cases $t$ and lt waves are excited. Since in this case both the resonance and growth rate conditions require $k_{\perp}>0$, we believe that this kind of excitation can explain the "cone" type of pulsar radio emission (Rankin 1983a).

In the subsequent papers in this volume (Kazbegi, Machabeli, and Melikidze 1992a, Kazbegi, Machabeli, and Melikidze 1992b) we will consider the problems of subpulse drift and circular polarization.

Acknowledgments: We would like to thank Dr. J. Gil for reading the manuscript, for valuable discussions and for help with preparing final version of this paper. 